\title{
TRATAMENTO DE SUPERFÍCIE E RESISTÊNCIA DE UNIÃO ENTRE PINO DE FIBRA DE VIDRO E A RESINA COMPOSTA
}

\section{SURFACE TREATMENT TYPE AND UNION RESISTANCE BETWEEN FIBERGLASS POSTS AND COMPOSITE RESIN}

\author{
Antonio Lucas Castro Pimentel', Rana de Brito Granja ${ }^{2}$, \\ Verônica Pereira Tschelakow ${ }^{3}$, Roberta Maria Novis ${ }^{4}$, Blanca Liliana Torres Léon ${ }^{5}$ \\ 'Graduado em odontologia. Residente em Cirurgia e Traumatologia Buco-Maxilo-Facial - HGRS/HGC/HGESF/EBMSP. \\ Salvador, Bahia, Brasil. lucas6591@gmail.com \\ ${ }^{2}$ Autora para correspondência. Graduanda em Odontologia na Universidade Federal da Bahia. Salvador, Bahia, Brasil. ranagranja@hotmail.com \\ ${ }^{3}$ Graduada em Odontologia pela Universidade Federal da Bahia. Salvador, Bahia, Brasil. veronica.thg@hotmail.com \\ ${ }^{4}$ Mestre em Prótese Dentária. Docente na Faculdade Regional da Bahia. Salvador, Bahia, Brasil. rmcnovis@gmail.com \\ ${ }^{5}$ Doutora em Clinica Odontológica. Docente na Universidade Federal da Bahia e na Escola Bahiana de Medicina e Saúde Pública. \\ Salvador, Bahia, Brasil. blalitole@hotmail.com
}

RESUMO I Objetivo: O objetivo deste trabalho foi avaliar a influência de diferentes métodos de tratamento da superfície em pinos de fibra de vidro com relação à sua força de união com a resina composta. Metódo: Foram utilizados vinte e quatro pinos pré-fabricados e divididos aleatoriamente em três grupos $(n=8)$ de acordo com o tratamento de superfície escolhido. Grupo Controle- tratamento de superfície com condicionamento ácido fosfórico a $37 \%$ e a silanização. Grupo 2 - condicionados com ácido fluorídrico a $10 \%$ e silanização. Grupo 3 - jateamento com óxido de alumínio por 10 segundos e silanização. Os corpos-de-prova foram obtidos com a utilização da matriz de aço inoxidável confeccionada para este estudo e foram mantidos em umidade absoluta a $37^{\circ} \mathrm{C}$ durante 48 horas para, então, serem submetidos ao teste de PUSH-OUT na máquina de teste. Os resultados foram avaliados pelo teste de Tukey com significância de 5\%. Resultados: Os resultados demostraram que o tratamento de jateamento de óxido de alumínio e silano (12,24 $\mathrm{MPa}$ ) (G3) apresentou diferença estatisticamente significante comparado com o tratamento de ácido fluorídrico e silano $(G 2)(8,03 \mathrm{MPa})(\mathrm{p}<0,05)$. O tipo de fratura predominante foi coesiva da resina composta $(68,75 \%)$ e apenas uma fratura mista $(2,08 \%)$ foi observada. Conclusão: Dessa forma, conclui-se que $\circ$ tratamento de jateamento de óxido de alumínio e silano pode ser utilizado como tratamento de superfície nos pinos de fibra de vidro.

\begin{abstract}
Objective: The objective of this work was to evaluate the influence of different methods of surface treatment on glass fiber posts with respect to their bond strength with the composite resin. Method: Twenty-four pre-fabricated posts were randomly divided into three groups $(n=8)$ according to the chosen surface treatment. Control Group- surface treatment with $37 \%$ phosphoric acid etching and silanization. Group 2 - conditioned with 10\% fluoridric acid and silanization. Group 3 - sandblasting for 10 seconds and silanization. The specimens were obtained using the stainless steel matrix made for this study and were maintained in absolute humidity at $37^{\circ} \mathrm{C}$ for 48 hours and then subjected to the PUSH-OUT test on the test machine. Results: The results showed that the aluminium oxide blasting treatment of aluminum and silane (12.24 MPa) (G3) had statistically significant difference when compared to the treatment of hydrofluoric acid and silane (G2) (8.03 MPa) $(p<0$, $05)$. The predominant fracture type was cohesive of the composite resin (68.75\%) and only a mixed fracture $(2.08 \%)$ was observed. Conclusions: Thus, it is concluded that the blasting treatment of aluminum oxide and silane can be used as a surface treatment on the glass fiber post.
\end{abstract}

Keywords: Fiber posts, bond strength, composite resins.

Palavras-Chave: Pinos dentários, resistência ao cisalhamento, resinas compostas. 


\section{INTRODUÇÃO}

Dentes tratados endodonticamente apresentam um desafio durante a sua reabilitação, pois a perda de tecido mineral devido à cárie, restaurações defeituosas, fraturas e ao próprio acesso endodôntico são fatores que dificultam o sucesso da restauração dessas unidades. Dessa forma, existe a necessidade de reconstruir a porção coronária para restabelecer o dente como elemento funcional e permanente do sistema estomatognático. Para garantir adequada retenção ao canal radicular, faz-se necessária a utilização de pinos nestas restaurações. Para tanto, pinos fabricados com fibra de carbono e reforçados com resina epóxi foram desenvolvidos no início dos anos $90^{\prime}$.

A restauração de dentes tratados endodonticamente, com a utilização de pinos intrarradiculares reforçados, tem chamado à atenção de um número crescente de cirurgiões dentistas. Atualmente, a utilização de materiais com módulo de elasticidade e características mecânicas mais próximas a da dentina vem crescendo na fabricação dos pinos. O progresso acerca da tecnologia empregada nos pinos visando estrutura, forma, e propriedades ópticas, devido ao aumento da demanda na indústria odontológica por materiais estéticos, levou ao desenvolvimento de pinos "metal-free", onde a zircônia, o quartzo e a fibra de vidro são os materiais mais comumente utilizados ${ }^{2}$.

Enquanto os pinos metálicos apresentam uma maior tendência a produzirem fraturas na região apical da raiz, pinos de fibra normalmente apresentam as fraturas na porção mais coronal da raiz, tornando a reestruturação da falha mais fácil. Os pinos de fibra de vidro estão se tornando cada vez mais populares em relação aos pinos de zircônia, pois, além de apresentar boa propriedade óptica, apresentam uma maior capacidade de adesão à dentina radicular ${ }^{3}$. Estudos in vivo vêm demonstrando que a união entre o complexo "dentina radicular pino - núcleo de preenchimento" é essencial para o sucesso de uma restauração retida com pinos ${ }^{4,5}$.

Para melhorar a forca de união entre os diferentes tipos de pinos e a resina composta, muitos tratamentos de superfície foram desenvolvidos.
Existem desde os tratamentos químicos, como a utilização de peróxido de hidrogênio, silano, e ácido fosfórico, aos tratamentos mecânicos, como - jateamento da superfície do pino com pequenas partículas de prata.

O silano é comumente utilizado como agente de tratamento de superfície, pois apresenta grande capacidade de unir partículas de fibra de vidro ao material restaurador ${ }^{2,4}$. Entretanto, existe controvérsia quanto à sua eficácia, já que foi relatado uma maior resistência de união após silanização², enquanto outras pesquisas, relatam a ausência de diferença estatística significativa entre pinos silanizados e não silanizados ${ }^{3}$.Outra opção de tratamento de superfície é o jateamento com óxido de alumínio, que cria retenções na superfície dos pinos de fibra para a resina composta. ${ }^{6}$ Entretanto, apesar de provocar trincas na superfície dos pinos, foi relatado que o jateamento não produz qualquer degradação das propriedades mecânicas dos pinos de fibra ${ }^{4}$.

As falhas mais comuns nas restaurações diretas ou indiretas associadas a pinos de fibra são o deslocamento da resina composta do núcleo ou da reconstrução completa do núcleo (pino/cimento resinoso /núcleo de resina) ${ }^{7}$. Assim, é de fundamental importância para $\circ$ cirurgião-dentista aprofundar $\circ$ conhecimento sobre as diversas formas de tratamento de superfície dos pinos para assim eleger o melhor tratamento e garantir um melhor prognóstico. Com base nisso, o objetivo deste trabalho foi avaliar a influência de diferentes métodos de tratamento da superfície em pinos estéticos de fibra de vidro em relação à sua força de união com a resina composta, e avaliar os tipos de fraturas entre o pino de fibra de vidro e à resina composta apos o teste de resistência ao cisalhamento por extrusão (PUSH-OUT).

\section{MATERIAIS E MÉTODOS}

\section{Materiais}

Os Materiais utilizados para $\circ$ presente estudo encontram-se listados na Tabela 1. 
Tabela 1. Materiais utilizados na pesquisa

\begin{tabular}{|c|c|c|c|c|}
\hline MATERIAL & $\begin{array}{c}\text { NOME } \\
\text { COMERCIAL }\end{array}$ & FABRICANTE & LOTE & VALIDADE \\
\hline $\begin{array}{c}\text { Agente de União } \\
\text { Silano }\end{array}$ & Prosil & FGM & 300515 & Maio 2017 \\
\hline $\begin{array}{l}\text { Condicionador } \\
\text { Ácido Fosfórico }\end{array}$ & Condac $37 \%$ & FGM & 170516 & Maio 2018 \\
\hline $\begin{array}{c}\text { Pino de Fibra de } \\
\text { Vidro }\end{array}$ & WhitePost DC & FGM & 061115 & $\begin{array}{c}\text { Novembro } \\
2020\end{array}$ \\
\hline $\begin{array}{c}\text { Resina Composta } \\
\text { Nanohíbrida }\end{array}$ & Opallis & FGM & 030914 & $\begin{array}{l}\text { Setembro } \\
2017\end{array}$ \\
\hline $\begin{array}{l}\text { Jateamento de } \\
\text { oxido de aluminio }\end{array}$ & $\begin{array}{l}\text { Oxido de } \\
\text { Alumínio }\end{array}$ & BIO-ART & $x$ & $x$ \\
\hline Acido Fluoridrico & $\begin{array}{c}\text { Condac Porcelana } \\
10 \%\end{array}$ & FGM & 030516 & Maio 2018 \\
\hline
\end{tabular}

\section{Tratamentos de superfície}

Para a realização deste trabalho foram utilizados um total de 24 pinos de fibra de vidro "Whitepost DC-E" (FGM, Joinville, SC, Brasil) tronco-cônicos, com comprimento de $18 \mathrm{~mm}$, secção transversal cilíndrica da maior extremidade de $2,2 \mathrm{~mm}$ e, da menor extremidade, de $1,05 \mathrm{~mm}$, sem curvaturas, trincas ou rachaduras (Figura 1). A porção cilíndrica do pino $(10 \mathrm{~mm}$ de altura) foi a região de escolha para receber os tratamentos de superfície, uma vez que toda a sua extensão apresentava as mesmas dimensões. Os pinos foram divididos aleatoriamente em três grupos (Tabela 2), contendo 8 pinos cada: G1(grupo controle), G2 (tratamento com ácido fluorídrico - HF) e G3(tratamento com jateamento de oxido de alumínio). Todos os pinos foram previamente imersos em álcool $70 \%$ durante 60 segundos.

Tabela 2. Disposição de Grupos, tratamento de superfície instituído e número de pinos por grupo.

\begin{tabular}{c|c|c}
\hline GRUPO & $\begin{array}{c}\text { TRATAMENTO DE } \\
\text { SUPERFÍCIE }\end{array}$ & $N^{\circ}$ DE PINOS \\
\hline G1 & $\begin{array}{c}\text { ÁCIDO FOSFÓRICO 37\% } \\
+ \text { SILANO }\end{array}$ \\
\hline G2 & ÁCIDO FLUORÍDRICO + \\
& SILANO & 8 \\
\hline G3 & JATEAMENTO + SILANO & 8 \\
\hline
\end{tabular}

No grupo controle $(G 1)$, os pinos foram tratados com ácido fosfórico a $37 \%$ (Condac - FGM, Joinvile, SC, Brasil) durante 30 segundos (Figura 2). Em seguida, foram lavados pelo mesmo tempo da aplicação do ácido, secados e receberam aplicação de Silano (Prosil - FGM, Joinville, SC, Brasil) (Figura 3) por 60 segundos, com auxílio de um microbrush. Cada pino foi posto em um Pote Dappen de vidro, durante 5 minutos, com o objetivo de obter uma secagem completa.

No grupo $G 2$, os pinos foram tratados com ácido fluorídrico (HF) a 10\% (Condac - FGM, Joinvile, 
SC, Brasil) (Figura 4) durante 30 segundos, além do Silano (Prosil - FGM, Joinville, SC, Brasil) por 60 segundos.

No grupo $G 3$, os pinos foram tratados por jateamento com micropartículas $(50 \mu \mathrm{m})$ de óxido de alumínio (Al2O3) durante 10 segundos à uma distancia de $10 \mathrm{~mm}$, sob pressão de 100 libras, por 20 segundos, usando um aparelho de jateamento
(Precision 1 - Essence Dental, Araraquara, SP, Brasil). $O$ pino foi girado manualmente para garantir que toda superfície fosse jateada. Após o jateamento, os pinos foram lavados vigorosamente com spray de ar/água por 60 segundos, para eliminação de resíduos de óxido de alumínio. Posteriormente, os pinos foram secos e receberam aplicação de Silano (Prosil - FGM, Joinville, SC, Brasil) por 60 segundos, com auxílio de um microbrush.

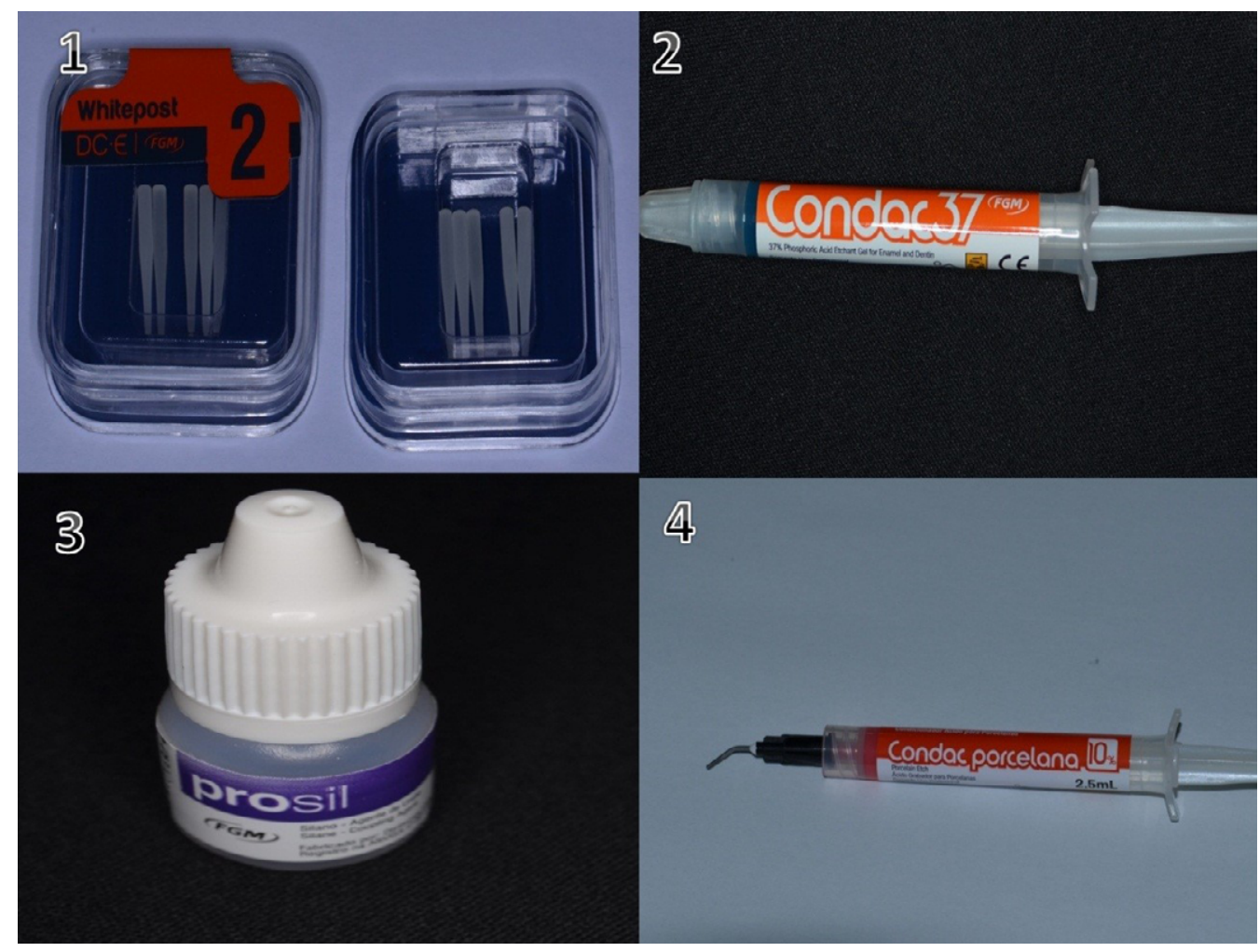

Figura 1, 2, 3 e 4

\section{Confecção dos corpos-de-prova}

Para confeccionar os corpos-de-prova, foi utilizada uma matriz metálica de aço bipartida, contendo um orifício cilíndrico central principal de $10 \mathrm{~mm}$ de altura e $5 \mathrm{~mm}$ de diâmetro e um orifício secundário de $2 \mathrm{~mm}$ de altura e diâmetro (Figura 5).

Para confecção das amostras foi necessário aplicar uma fina camada de vaselina sólida (Rioquímica, São José do Rio Preto, SP, Brasil) no interior da matriz, com ajuda de um pincel (KOTA, Cotia, SP, Brasil) e, a seguir, foi inserido uma lâmina de cera utilidade (Lysanda, São Paulo, SP, Brasil) para imobilização do pino no orifício menor. Após a finalização do tratamento de superfície dos pinos (como foi descrito anteriormente), os mesmos, foram adaptados com auxílio de uma pinça clínica à matriz metálica.

Posteriormente, preencheu-se por incrementos de 2 $\mathrm{mm}$ no interior da matriz, a resina Opallis (FGM, Joinville, SC, Brasil). A fotoativação foi realizada no sentido ocluso-apical com o fotopolimerizador Ultralux EL (Dabi Atlante) com intensidade $500 \mathrm{mw} / \mathrm{cm}^{2}$ durante 40 segundos. Foi repetido - preenchimento e a fotopolimerização mais quatro vezes, totalizando cinco incrementos de 2 $\mathrm{mm}$ cada. Após $\circ$ preenchimento total os corpos de prova foram removidos da matriz (Figura 6) e foram seccionados transversalmente com discos diamantados bilaminados (Fava, São Paulo, SP, Brasil) em três amostras de aproximadamente 3 
$\mathrm{mm}$ de altura cada (Figura 7). Para realização do seccionamento, cada corpo de prova foi fixado com godiva (Kerr, São Paulo, SP, Brasil) dando origem a três amostras. Essas amostras correspondem ao terço apical, terço médio e terço cervical. Foi descartado o terço apical devido ao contato do pino com a cera utilidade, utilizada para conferir maior estabilidade entre o pino e a matriz.

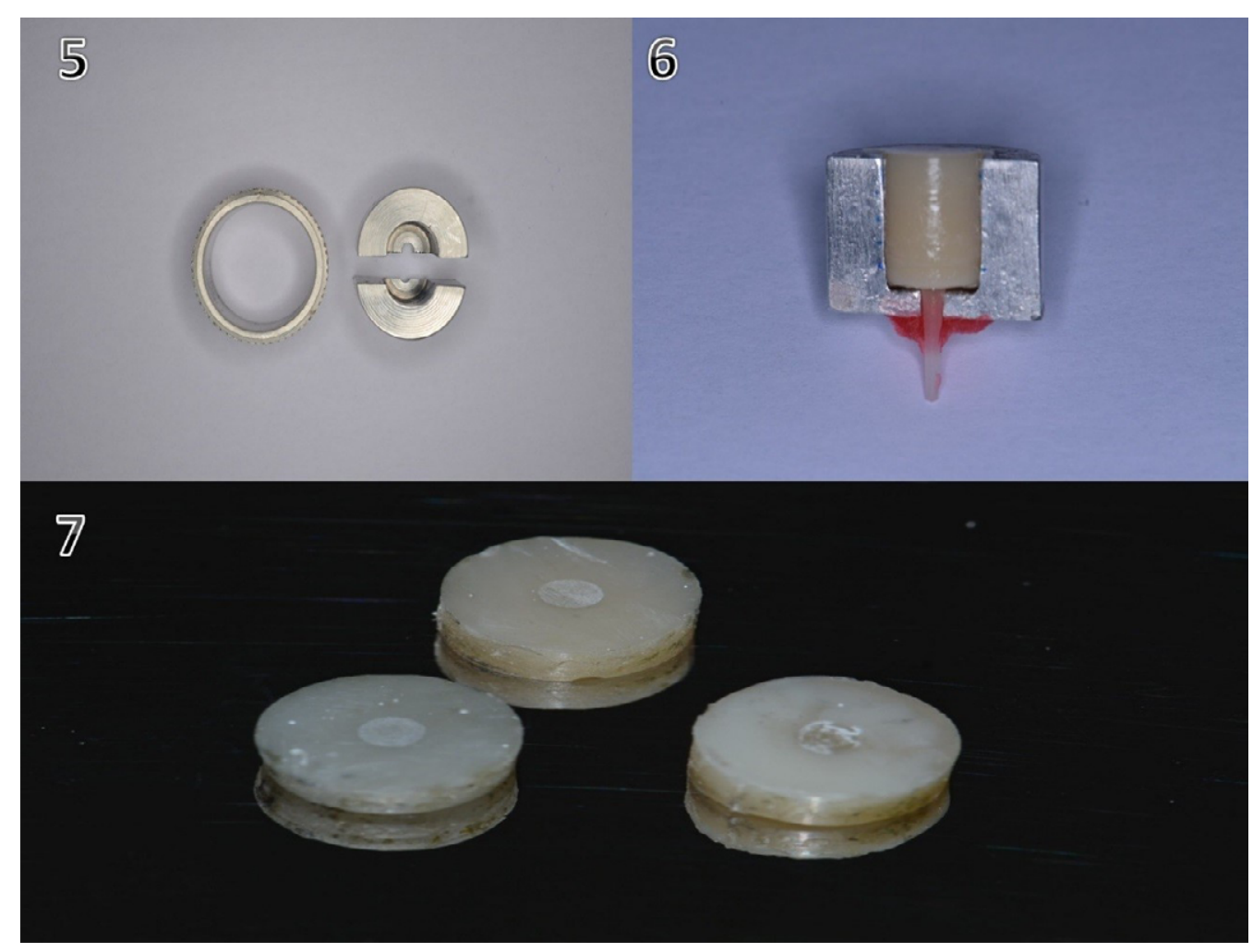

Figura 5, 6 e 7

As amostras tiveram sua altura mensurada com - auxílio de um paquímetro digital (JOMARCA, São Paulo, SP, Brasil). Após secção, realizou-se o acabamento dos espécimes com discos de lixa (TDV, Pomerode, SC, Brasil) para a resina composta na ordem sequenciada conforme o fabricante.

\section{Ensaio mecânico de cisalhamento por extrusão "push out"}

Cada amostra, foi posicionado no dispositivo desenvolvido especificamente para este teste, constituído por base metálica em aço inoxidável com altura de $10 \mathrm{~mm}$, diâmetro interno de $10 \mathrm{~mm}$, com um orifício de 2 milímetros $(\mathrm{mm})$ na região central (Figura 8). As amostras foram submetidas ao teste Push-Out, realizado em Máquina de Ensaio Universal (EMIC), onde foi avaliada a força de extrusão por cisalhamento dos pinos (Figura 9). Assim, foi possível avaliar qual dos diferentes meios de tratamento de superfície forneceu uma maior união entre pino e resina. Para determinar a resistência de união em Mega Pascal (Mpa), os valores da força máxima em Newton $(N)$ foram divididos pela área da interface de união. $O$ cálculo foi feito por meio da seguinte fórmula: 2 rh, onde $r$ é o raio do pino medindo 1 $\mathrm{mm}$, e h a altura da amostra8. 


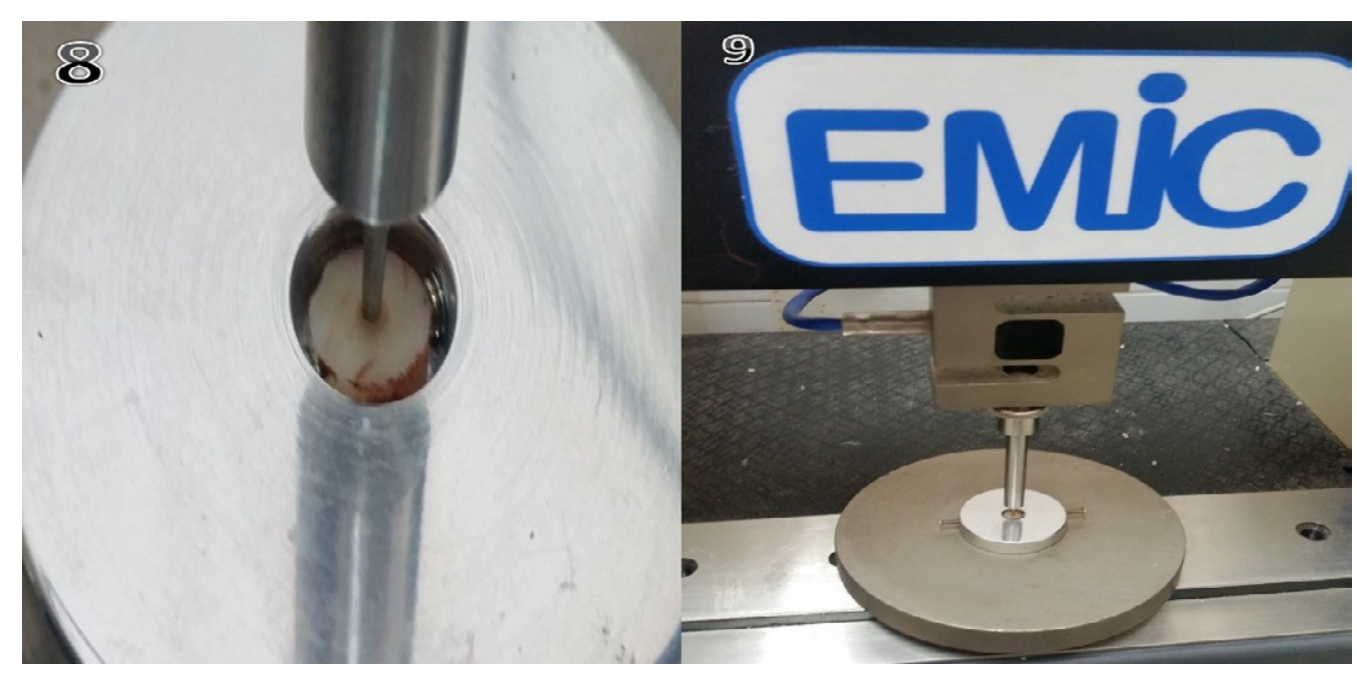

Figura 8 e 9

Após o teste Push-Out, foi avaliado o tipo de fratura, utilizando um microscópio óptico com 30x de aumento (Maquira, Maringá, PR, Brasil). As fraturas foram classificadas como: Tipo I - Fratura adesiva: quando a falha ocorre na interface entre pino de fibra e resina composta; Tipo II - Fratura coesiva em resina: quando a falha ocorre exclusivamente no interior da resina composta; Tipo III - Fratura coesiva no pino: quando a falha ocorre exclusivamente no interior da secção do pino de fibra; Tipo IV - Fratura mista: combinação das falhas anteriores?.

\section{Análise estatística}

Os resultados foram submetidos à análise de variância e as médias foram comparadas utilizando o teste de Tukey com $5 \%$ de significância.

\section{RESULTADOS}

Na Tabela 3, a análise de variância mostrou indícios $(p<0,05)$ de diferenças entre as médias da resistência de cisalhamento verdadeiras de pelo menos, dois dentre os grupos estudados.

Tabela 3. Análise de variância para teste dos efeitos sobre a Resistência (MPa) calculado com base em modelo linear generalizado misto para dados com medidas repetidas.

\begin{tabular}{lccccc}
\hline & & & Graus de liberdade & \multicolumn{2}{c}{ Teste de F } \\
& Efeito & Numerador & Denominador & Estatística & Valor-p \\
\cline { 1 - 4 } Grupo & 2 & 21 & 3,82 & 0,0386 \\
Terço & 1 & 21 & 3,00 & 0,0980 \\
Grupo * Terço & 2 & 21 & 0,36 & 0,7023 \\
\hline
\end{tabular}

A Tabela 4 e Figura 10 comparam as médias e o desvio-padrão (SD) da resistência ao cisalhamento nos três grupos: Gl (grupo controle), G2 (ácido fluorídrico e Silano) e G3 (jateamento de oxido de alumínio e silano). Na mesma Tabela e Figura, o grupo G3 (12,24 MPa) mostrou os maiores valores que o grupo G2 $(8,03 \mathrm{MPa})$. 
Tabela 4. Teste de Tukey, média, desvio padrão e limites de confiança da média (95\%) de Resistência (MPa). Médias com letras iguais não diferem entre si no nível de significância de $5 \%$.

\begin{tabular}{|c|c|c|c|c|c|}
\hline \multirow[b]{2}{*}{ Grupo } & \multirow{2}{*}{$\begin{array}{c}\text { Teste de Tukey } \\
(\square=0,05)\end{array}$} & \multirow[b]{2}{*}{ Média } & \multirow{2}{*}{$\begin{array}{l}\text { Desvio } \\
\text { padrão }\end{array}$} & \multicolumn{2}{|c|}{ Limite de confiança $(95 \%)$} \\
\hline & & & & superior & inferior \\
\hline G3 & A & 12,24 & 3,89 & 14,31 & 10,16 \\
\hline G1 & A B & 10,60 & 5,04 & 13,28 & 7,91 \\
\hline G2 & B & 8,03 & 4,12 & 10,22 & 5,83 \\
\hline
\end{tabular}

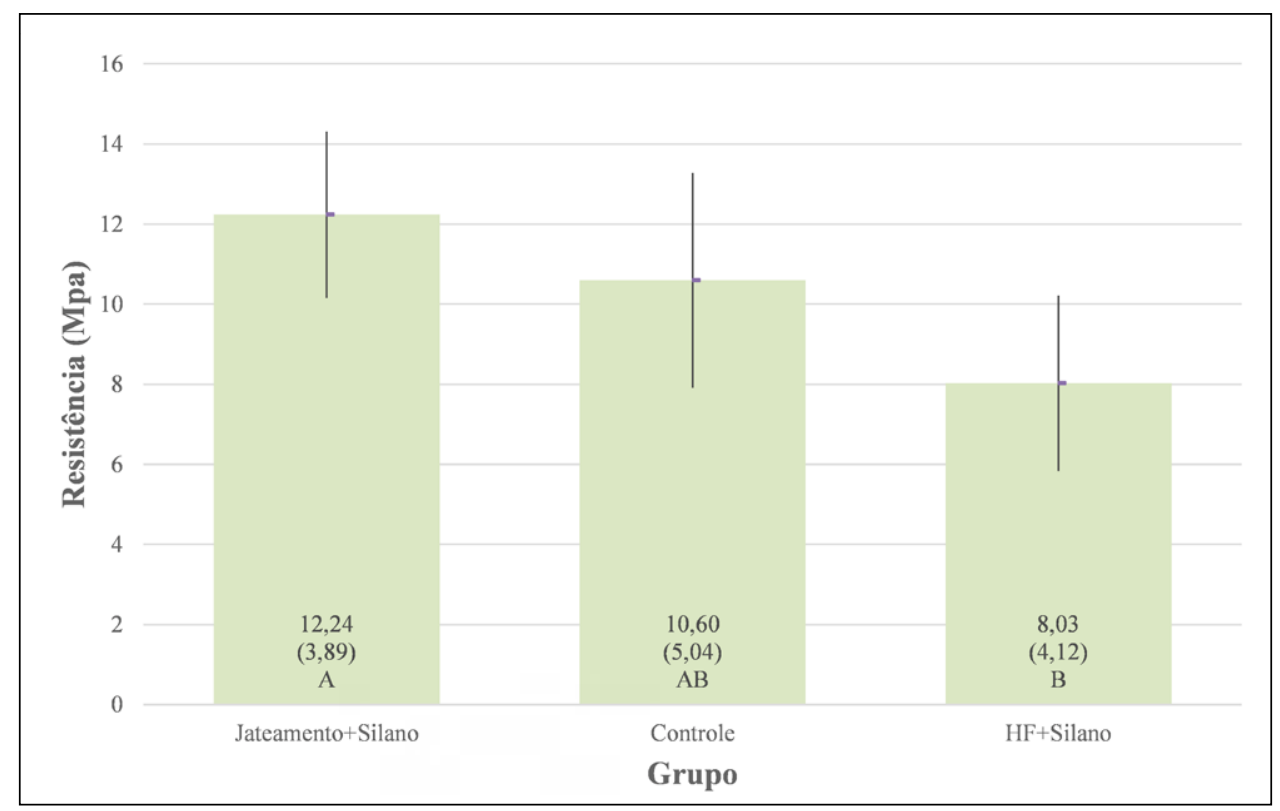

Figura 10

A Tabela 5, analisa a comparação das médias da resistência de cisalhamento (MPa) resultantes da combinação dos grupos e terços cervical e médio. Observa-se que tanto no terço cervical como no terço médio a maior média é observada no grupo $G 3$ e a menor média no grupo $G 2$, deixando o controle em posição intermediária a qual não difere dos extremos, os quais, por sua vez, diferem significativamente entre si.

Tabela 5. Média, desvio padrão e limites de confiança da média (95\%) de Resistência (MPa) nas combinações de grupos e terços.

\begin{tabular}{|c|c|c|c|c|c|}
\hline \multirow[b]{2}{*}{ Terço } & \multirow[b]{2}{*}{ Grupo } & \multirow[b]{2}{*}{ Média } & \multirow{2}{*}{$\begin{array}{l}\text { Desvio } \\
\text { padrão }\end{array}$} & \multicolumn{2}{|c|}{ Limite de confiança (95\%) } \\
\hline & & & & superior & inferior \\
\hline & G3 & 12,69 & 3,88 & 15,94 & 9,44 \\
\hline \multirow[t]{3}{*}{ Cervical } & G1 & 11,64 & 5,66 & 16,37 & 6,91 \\
\hline & G2 & 9,78 & 4,64 & 13,66 & 5,90 \\
\hline & G3 & 11,78 & 4,12 & 15,22 & 8,34 \\
\hline \multirow[t]{2}{*}{ Médio } & G1 & 9,55 & 4,46 & 13,28 & 5,83 \\
\hline & G2 & 6,27 & 2,78 & 8,60 & 3,95 \\
\hline
\end{tabular}

Com relação aos tipos de fratura, observa-se que o padrão de fratura (Tabela 6) mais recorrente é $\circ$ de fratura coesiva na resina (Tipo II) que ocorreu em 33 casos $(68,75 \%)$. Isso representa mais que o dobro do segundo tipo mais recorrente (Adesiva - Tipo II) observado em 14 casos $(29,17 \%)$. Juntos esses dois tipos 
respondem por $97,92 \%$ da amostra (47 de 48 casos) o que nos permite deduzir que a fratura mista (Tipo IV) seja relativamente rara, posto que ocorreu em apenas uma amostra $(2,08 \%)$.

Tabela 6. Frequências e porcentagens, simples e acumuladas de ocorrências das categorias e teste de qui-quadrado para igualdade de proporções.

\begin{tabular}{|c|c|c|c|c|}
\hline \multirow[b]{2}{*}{ Variáveis / categorias } & \multicolumn{2}{|c|}{ Simples } & \multicolumn{2}{|c|}{ Acumulada } \\
\hline & Frequência & Porcentagem & Frequência & Porcentagem \\
\hline \multicolumn{5}{|c|}{ Padrão de fratura ( $\square^{2}: 32,37$ - GL: 2 - p:0,0001) } \\
\hline Adesiva & 14 & 29,17 & 14 & 29,17 \\
\hline Coesiva da resina & 33 & 68,75 & 47 & 97,92 \\
\hline Mista & 1 & 2,08 & 48 & 100,00 \\
\hline \multicolumn{5}{|c|}{ Terço ( $\square^{2}: 0,00$ - GL: 1 - p:1,0000) } \\
\hline Cervical & 24 & 50,00 & 24 & 50,00 \\
\hline Médio & 24 & 50,00 & 48 & 100,00 \\
\hline \multicolumn{5}{|c|}{ Grupo ( $\left.\square^{2}: 0,00-G L: 2-p: 1,0000\right)$} \\
\hline Controle & 16 & 33,33 & 16 & 33,33 \\
\hline $\mathrm{HF}+$ Silano & 16 & 33,33 & 32 & 66,66 \\
\hline Jateamento+Silano & 16 & 33,33 & 48 & 100,00 \\
\hline \multicolumn{5}{|c|}{ Classe de resistência ( $\left.\square^{2}: 0,00-G L: 1-p: 1,0000\right)$} \\
\hline Abaixo da mediana & 24 & 50,00 & 24 & 50,00 \\
\hline Acima da mediana & 24 & 50,00 & 48 & 100,00 \\
\hline
\end{tabular}

$\square^{2}$ : Qui-quadrado; GL: graus de liberdade; p:Valor-p Ho: igualdade de proporções.

Os Grupos G1 (Controle) e Grupo G3 (Jateamento + Silano) apresentam um comportamento similar em relação à distribuição de casos entre os padrões de fratura já que em ambos, mais de $80 \%$ das fraturas ocorreram dentro do padrão "Coesiva da resina". Entretanto, no Grupo 2 (HF + Silano), apresentou uma maior prevalência de fraturas de tipo adesivas (60\%). (Tabela 7).

Tabela 7. Tabela de contingência e teste de qui-quadrado de razão de verossimilhança para avaliação da associação entre padrão de fratura e demais variáveis categóricas do estudo.

\begin{tabular}{|c|c|c|c|}
\hline \multirow[b]{2}{*}{ Variáveis / categorias } & \multicolumn{2}{|c|}{ Padrão de fratura } & \multirow[b]{2}{*}{ Total } \\
\hline & Adesiva & Coesiva da resina & \\
\hline \multicolumn{4}{|c|}{ Terço (G²:0,54 - GL: 1 - p:0,4630) } \\
\hline Cervical & $8(34,78)$ & $15(65,22)$ & $23(100,00)$ \\
\hline Médio & $6(25,00)$ & $18(75,00)$ & $24(100,00)$ \\
\hline \multicolumn{4}{|c|}{ Grupo $\left(G^{2}: 9,56-G L: 2-p: 0,0084\right)$} \\
\hline G1 & $2(12,50)$ & $14(87,50)$ & $16(100,00)$ \\
\hline G2 & $9(60,00)$ & $6(40,00)$ & $15(100,00)$ \\
\hline G3 & $3(18,75)$ & $13(81,25)$ & $16(100,00)$ \\
\hline \multicolumn{4}{|c|}{ Classe de resistência (G²:6,33-GL: $1-p: 0,0118)$} \\
\hline Abaixo da mediana & $11(45,83)$ & $13(54,17)$ & $24(100,00)$ \\
\hline Acima da mediana & $3(13,04)$ & $30(86,96)$ & $23(100,00)$ \\
\hline
\end{tabular}

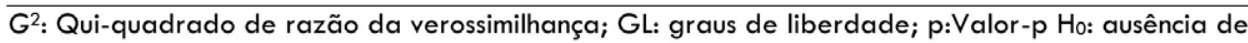
associação entre padrões de fratura e variáveis de controle. 


\section{DISCUSSÃO}

Os tratamentos de superfície podem ser realizados de três formas: métodos químicos (aplicação de ácidos), métodos físicos (como o jateamento) e associação de métodos físicos e químicos. Estes tratamentos têm como objetivo promover uma maior rugosidade de superfície e maior interação química entre a interface de união dos compostos resinosos e pinos de fibra de vidro ${ }^{7}$.

Diversos estudos observaram que, o condicionamento com ácido fluorídrico dissolve os componentes vítreos e cristalinos, alterando de forma significativa a morfologia superficial dos pinos de fibra de vidro, promovendo irregularidades representadas por microporos retentivos, fendas e sulcos ${ }^{7,10,11}$. Dessa forma, tem a capacidade de aumentar a molhabilidade do composto resinoso aplicado a superfície do pino., ${ }^{70,12,13} \bigcirc$ ácido fluorídrico é comumente utilizado para o tratamento de superfície de cerâmicas, pois este procedimento, seguido da aplicação de silano, produz união clinicamente aceitável da resina composta para cerâmica à base de Sílica ${ }^{7}, 11,13,14$. Por outro lado, foi constatado que o ácido fosfórico, quando empregado como método de tratamento de superfície, proporciona pouca alteração superficial na estrutura dos pinos de fibra de vidro, atuando de maneira mais eficiente na limpeza superficial do mesmo ${ }^{5}$.

Baseado nos resultados encontrados, o tratamento de superfície por jateamento com óxido de

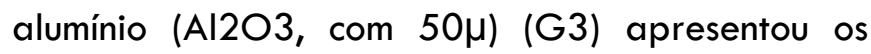
maiores valores de resistência de união entre 0 pino de fibra de vidro e resina composta $(12,24$ $\mathrm{MPa}$ ) quando comparado ao tratamento de ácido fluorídrico (grupo G2). A maior força de união pode ser explicada pela criação de micro retenções superficiais. Este condicionamento, por ser realizado sob pressão, e com partículas de óxido de alumínio com alta dureza, ao impactarem sobre a superfície do pino de fibra de vidro, causam alterações morfológicas ${ }^{7}$. Posteriormente, a aplicação do agente silano torna a superfície mais reativa à resina ${ }^{15}$. Nos estudos de Goracci et al. ${ }^{2}$; Radovic et al. ${ }^{16}$ e Soares et al. $^{17}$, constataram o efeito positivo na aplicação de silano, como agente promotor de adesão na interface pino e resina composta. Os silanos são compostos orgânicos que contêm silício (Si), podendo ser bifuncionais, ou seja, ter duas extremidades reativas. A parte orgânica funcional (p. ex., vinila, alila, amina ou isocianato) pode reagir com uma matriz orgânica.

Por outro lado, os menores valores de resistência de união que foram encontrados para o grupo G2 (ácido fluorídrico) $(8,03 \mathrm{MPa})$, pode estar associado, a que $\circ$ ácido, pode ser muito agressivo à superfície do pino, destruiu de forma não seletiva a camada mais externa da matriz, causando alterações físicomecânicas significativas na estrutura do pino $8,18,19,20$. Estes resultados concordam com 0 trabalho de Valandro ${ }^{15}$ et al. Os autores avaliaram os tratamentos de superfície para pinos de fibra de quartzo em 3 grupos, utilizando: (G1) condicionamento com ácido fosfórico $32 \%$ (1 $\mathrm{min}$ ) + silano, (G2) condicionamento com ácido fluorídrico 10\% (1 min) + silano, e (G3) Sistema Cojet (jateamento com partículas de $\mathrm{Al} 2 \mathrm{O} 3$ $30 \mu \mathrm{m}$ modificadas por $\mathrm{SiOx}+$ silano), variando deste estudo apenas quanto à concentração do ácido fosfórico e ao tamanho das partículas de óxido de alumínio. Os valores de resistência adesiva foram afetados pelo tratamento de superfície, sendo o tratamento (G3) significativamente maior que $\circ$ tratamento $(G 2)$ e este significativamente maior que $\circ(G 1)$. Comprovando que a maior rugosidade superficial proveniente do jateamento, quando comparado a métodos de tratamento de superfície químicos, proporciona uma maior adesividade entre o pino e o composto resinoso.

O jateamento com óxido de alumínio é utilizado a fim de aumentar a rugosidade da superfície pela remoção parcial da matriz epóxi do pino ${ }^{11}$. Embora alguns estudos demonstrem que esta remove apenas a parte epóxica do pino, outros estudos mostram que este tratamento pode levar a danos nas fibras. Os resultados conflitantes podem estar associados aos diferentes tempos de aplicação, ao tamanho das partículas de óxido de alumínio e à pressão e à distância do aplicador à superfície do pino. O fato é que, a superioridade na rugosidade superficial dos pinos tratados fisicamente através do jateamento confere uma maior união entre os substratos resinosos e o pino ${ }^{8,10,21}$. Dessa forma, a utilização clínica de pinos tratados através do jateamento seguido de aplicação de silano deverá proporcionar uma maior longevidade do tratamento ${ }^{6,13}$.

Em 2003, Monticelli et al. ${ }^{4}$ publicaram uma revisão de literatura onde investigaram estudos sobre 
condicionamento de superfície de pinos, visando avaliar diferentes métodos de aumento da resistência adesiva entre pino e compósito. Os dados coletados pelos autores foram divididos em três categorias: tratamentos que resultam em adesão química entre pino e compósito; tratamentos que objetivam tornar a superfície do pino mais rugosa (jateamento ou condicionamento) ou combinação de métodos micro-mecânicos e químicos utilizando ambos os métodos citados acima. Os autores conclúram que a combinação entre retenção química e micromecânica na superfície do pino pode ser a alternativa mais indicada para uma melhor adesão.

Clinicamente, espera-se que a união entre pino e compósito resinoso seja capaz de resistir às cargas oclusais do paciente em função. Balbosh et al.6 avaliaram $\circ$ envelhecimento artificial relacionado à retenção de pinos de fibra em quatro diferentes formas de tratamento de superfície de pinos de fibra de vidro. Os autores concluíram que, após 300.000 ciclos sob uma forca de $30 \mathrm{~N}$, o grupo com jateamento de óxido de alumínio apresentou uma maior resistência ao teste de tração. É necessário enfatizar que, clinicamente, esta força de união verificada entre o pino de fibra de vidro e os compostos resinosos é de extrema importância, uma vez que é necessário correlacionar tais valores com o padrão de forças mastigatórias de cada indivíduo.

No presente estudo, constatou-se uma prevalência de fraturas coesivas da resina, tanto no Grupo Controle G1 quanto no Grupo 3. A fratura coesiva da resina caracteriza-se por apresentar uma deformidade inerente ao composto resinoso, que se caracteriza por defeitos como trincas e fraturas nesse material. ${ }^{19}$ Clinicamente, este seria o tipo de fratura que proporcionaria menor prejuízo ao paciente, pois não afetará a estrutura do remanescente dental e nem mesmo do pino de fibra de vidro. A correção desta fratura se dará de maneira mais simples, quando comparada às demais fraturas, pois é necessária apenas a reconstrução da restauração em resina ou substituição da mesma.

Por outro lado, também foi constatado neste estudo, que a quantidade de fraturas adesivas no $G 2$ foi consideravelmente maior que no $G 1$ e no $G 3$. Isto pode estar associado, a que o ácido fluorídrico condicionou não seletivamente as fibras e a matriz, danificando a microestrutura dos pinos, o que possivelmente levou a uma alteração na força de união adesiva entre o pino de fibra de vidro e a resina composta. ${ }^{5}$ Clinicamente, trata-se da fratura mais danosa ao paciente, o que acarretará em prejuízos na força de união entre o pino e a resina composta.

\section{CONCLUSÃO}

Baseado nos resultados obtidos neste estudo, concluise que, o jateamento com óxido de alumínio por 10 segundos, pode ser utilizado como tratamento de superfície, nos pinos de fibra de vidro. Também foi observado que, o maior número de fraturas encontradas foram as do tipo "Coesiva da Resina", no grupo controle (G1) e no grupo de jateamento de óxido de alumínio (G3).

Assim, futuras pesquisas, clínicas e laboratoriais, são necessárias para determinar a eficácia de diferentes tratamentos alternativos de superfície sobre os pinos de fibra, e no comportamento da resistência de união à resina composta.

\section{CONFLITOS DE INTERESSES}

Nenhum conflito financeiro, legal ou político envolvendo terceiros (governo, empresas e fundações privadas, etc.) foi declarado para nenhum aspecto do trabalho submetido (incluindo mas não limitandose a subvenções e financiamentos, conselho consultivo, desenho de estudo, preparação de manuscrito, análise estatística, etc).

\section{REFERÊNCIAS}

1. Duret B, Reynaud M, Buret F. A new concept of coronoradicular reconstruction, the Composipost. Chir Dent Fr. 1990;60(542):69-77.

2. Goracci C, Raffaelli O, Monticelli F, Balleri B, Bertelli E, Ferrari $M$. The adhesion between prefabricated $F R C$ posts and composite resin cores: Microtensile bond strength with and without post-silanization. Dent Mater. 2005;21(5):43744. doi: 10.1016/i.dental.2004.07.012

3. Perdigão J, Gomes G, Lee IK. The effect of silane on the bond strengths of fiber posts. Dent Mater. 2006;22(8):752-8. doi: $10.1016 /$ i.dental.2005.11.002 
4. Monticelli F, Grandini S, Goracci C, Ferrari M. Clinical behavior of translucent fiber posts: A 2-year prospective study. Int J Prosthodont. 2003;16(6):593-6.

5. Vano M, Goracci C, Monticelli F, Tognini F, Gabriele $M$, Tay FR et al. The adhesion between fibre posts and composite resin cores: The evaluation of microtensile bond strength following various surface chemical treatments to posts. Int Endod J. 2006;39(1):31-9. doi: 10.1111/i.13652591.2005.01044.x

6. Balbosh A, Kern M. Effect of surface treatment on retention of glass-fiber endodontic posts. J Prosthet Dent. 2006;95(3):218-23. doi: 10.1016/i.prosdent.2006.01.006

7. Goracci C, Ferrari M. Current perspectives on post systems: A literature review. Aust Dent J.201 1;56(supl 1):77-83. doi: 10.1111/i.1834-7819.2010.01298.x

8. Marques JN, Gonzalez CB, da Silva EM, Pereira GDS, Simão RA, do Prado M. Análise comparativa da resistência de união de um cimento convencional e um cimento autoadesivo após diferentes tratamentos na superfície de pinos de fibra de vidro. Rev Odontol UNESP. 2016;45(2):1 21 -6. doi: 10.1590/1807-2577.18615

9. Andreatti FL. Influência do tipo de tratamento da superfície de pinos de fibra de vidro na resistência de união à resina composta [tese]. Florianópolis: Universidade Federal de Santa Catarina; 2011.

10. Novais VR, Júnior PCS, Rontani RMP, Correr-Sobrinho L, Soares CJ. Bond strength between fiber posts and composite resin core: influence of temperature on silane coupling agents. Braz Dent J. 2012;23(1):8-14. doi: 10.1590/S0103$\underline{64402012000100002}$

11. Baratieri LN. Abordagem restauradora de dentes tratados endodonticamente: pinos/núcleos e restaurações unitárias. In: Baratieri LN, Junior MS, Andrada MAC, Vieira LCC, Ritter AV, Cardoso AC. Odontologia restauradora: fundamentos e possibilidades. São Paulo: Santos; 2007. p. 619-71.

12. Rungruanganunt $P$, Kelly JR. Insights into "bonding" of all-ceramics influenced by cement, sandblasting and water storage time. Dent Mater. 201 2;28(9):939-44. doi: $10.1016 /$ i.dental.2012

13. De Boever JA, McCall WD, Holden S, Ash MM. Functional occlusal forces: An investigation by telemetry. J Prosthet Dent. $1978 ; 40(3): 326-33$.

14. Sultan SE, Korsiel AM, Kamel MS, Etman WM. Effect of different surface treatments on bond strength of fiber post to root dentin. Tanta Dent J. 2013;10(3):1 16-22. doi: $\underline{10.1016 / i . t d i .2013 .11 .003}$

15. Valandro LF, Baldissara $P$, Galhano GA, Melo RM, Mallmann A, Scotti R et al. Effect of mechanical cycling on the push-out bond strength of fiber posts adhesively bonded to human root dentin. Operative Dentistry. 2007;32(6),579-88. doi: $10.2341 / 06-165$

16. Radovic I, Monticelli F, Goracci C, Cury ÀH, Coniglio I, Vulicevic ZR et al. The effect of sandblasting on adhesion of a dual-cured resin composite to methacrylic fiber posts: Microtensile bond strength and SEM evaluation. J Dent. 2007;35(6):496-502. doi: 10.1016/i.jdent.2007.01.009

\section{Soares CJ, Soares PV, Santos-Filho PCF, Castro} CG, Magalhaes D, Versluis A. The Influence of Cavity Design and Glass Fiber Posts on Biomechanical Behavior of Endodontically Treated Premolars. J Endod. 2008;34(8):1015-9. doi: 10.1016/i.joen.2008.05.017

18. Ferrari M, Vichi A, Grandini S. Efficacy of different adhesive techniques on bonding to root canal walls: An SEM investigation. Dent Mater. 2001;17(5):422-9.

19. Faria MIA, Gomes EA, Messias DC, Filho JMS, Filho CBS, Paulino SM. Tensile strength of glass fiber posts submitted to different surface treatments. Braz Dent J. 2013;24(6):626-9. doi: $10.1590 / 0103-6440201302365$

20. Bordin D, Cavalcanti YW, Farina AP, Carlini-Júnior B, Cecchin D. Long-term effect of ethanol on the bond strength of fiber-glass posts to root dentin. Rev Odonto Cienc. 2014;29(2):35-9. doi: 10.15448/1980-6523.2014.2.12666

21. Matinlinna JP, Lassila LV, Ozcan M, Yli-Urpo A, Vallittu PK. An introduction to silanes and their clinical applications in dentistry. Int J Prosthodont. 2004;17(2):155-64. 